\title{
On the Photometric Accuracy of RHESSI Imaging and Spectrosocopy
}

\author{
Markus J. Aschwanden ${ }^{1}$, Thomas R. Metcalf ${ }^{1}$, Säm Krucker ${ }^{2}$, Jun \\ Sato $^{3}$, Andrew J. Conway ${ }^{4}$, Gordon J. Hurford ${ }^{2}$, and Ed, J. \\ Schmahl $l^{5,6}$ \\ 1) Lockheed Martin Advanced Technology Center, Solar 8 Astrophysics Laboratory, \\ Dept. L9-41, Bldg.252, 3251 Hanover St., Palo Alto, CA 94304, USA; e-mail: \\ aschwanden@lmsal.com, \\ 2) Space Sciences Laboratory, University of California at Berkeley, Berkeley, CA \\ 94720-7450; \\ 3 ) Dept. of Physics, Montana State University, Bozeman, MO 59717; \\ $\left.{ }^{4}\right)$ Dept. Physics and Astronomy, The Open University, Milton Keynes MK' 6 AA, \\ $U K$; \\ 5) Solar $\&$ Astrophysics Laboratory, NASA Goddard Space Flight Center, \\ Greenbelt, MD 20770, USA; \\ 6) Astronomy Dept, University of Maryland, College Park, MD 20742.
}

$2003 / \mathrm{Aug} / 14$

\begin{abstract}
We compare the photometric accuracy of spectra and images in flares observed with the Ramaty High Energy Solar Spectroscopic Imager (RHESSI) spacecraft. We test the accuracy of the photometry by comparing the photon fluxes obtained in different energy ranges from the spectral-fitting software SPEX with those fluxes contained in the images reconstructed with the Clean, MEM, MEMVis, Pixon, and Forward-fit algorithms. We quantify also the background fluxes, the fidelity of source geometries, and spatial spectra reconstructed with the five image reconstruction algorithms. We investigate the effects of grid selection, pixel size, field-of-view, and time intervals on the quality of image reconstruction. The detailed parameters and statistics are provided in an accompanying CD-ROM and web page. We find that Forward-fit, Pixon, and Clean have a robust convergence behavior and a photometric accuracy in the order of a few percents, while MEM does not converge optimally for large degrees of freedom (for large field-of-views and/or small pixel sizes), and MEM-Vis suffers in the case of time-variable sources. This comparative study documents the current status of the RHESSI spectral and imaging software, one year after launch.
\end{abstract}

Keywords: Sun : flares — Sun : hard X-rays

\section{INTRODUCTION}

The Reuven Ramaty High Energy Solar Spectroscopic Imager (RHESSI) was launched on 2002 Feb 5 and operated successfully through the first year, recording a total of $\approx 7500$ solar flares. At this time, after one year of the mission, most of the software has matured to be both reliable and accurate, and instrumental parameters have been improved

(c) 2018 Kluwer Academic Publishers. Printed in the Netherlands. 
from in-flight data to great precision. Thus, it is timely to conduct a quantitative comparison of the performance of the various available imaging algorithms, to compare the spatial and spectral features in the reconstructed images, and to cross-calibrate the photon fluxes extracted from images against those from the (non-imaging) spectral fitting software. Such a quantitative comparison will also be useful to provide estimates of uncertainties and systematic errors in spectral fitting and spectral inversions, as well as to serve as a reference for monitoring future changes in the instrumental parameters and software developments. All image reconstructions are performed here with the software version available in March 2003.

The RHESSI spacecraft and its mission are described in the overview article of Lin et al. (2002), the RHESSI spectrometer in Smith et al. (2002), and the RHESSI imaging concepts in Hurford et al. (2002). Further technical articles that may be of interest in the context of this study are on RHESSI data analysis software (Schwartz et al. 2002), on reconstruction of RHESSI images with forward-fitting (Aschwanden et al. 2002a), and on pixon image reconstruction (Metcalf et al. 1996). We choose for this comparative study as main event the flare of 2002 Feb 20, 11:06 UT, because this event was already subject of various analyses in a number of previous publications (Krucker \& Lin 2002, Sui et al. 2002, Vilmer et al. 2002, Brown et al. 2002, Aschwanden et al. 2002b, Kontar et al. 2002), as well as the two additional flares of 2002 May 18, 19:15 UT, and 2002 Jul 23, 00:30 UT.

\section{RESULTS OF DATA ANALYSIS}

The details of the data analysis are provided on the accompanying CDROM (or on the web-page http://www.lmsal.com/ aschwand/eprints/ 2003_photo/index.html). Here we summarize only the main results of this analysis, which includes a detailed parametric study of the 2002 Feb 20 flare (§A1-A11 on CD-ROM), as well as additional tests on flares with different source morphologies and flare positions, i.e., the singlesource flare from 2002 May 18 ( $\S \mathrm{B} 1$ on CD-ROM) and the complexsource X-ray flare of 2002 Jul 23 ( $\S \mathrm{C} 1$ on CD-ROM).

\subsection{Spectral Photometry (§A1 on CD-ROM)}

The photometry can be obtained from two different software codes in the RHESSI SolarSoftWare (SSW), either from the spectral fitting package SPEX (Schwartz et al. 2002; Smith et al. 2002), or from the RHESSI image reconstruction algorithms. Both codes use the same 
Table I. Ratios of image-integrated photon fluxes to the total spectral fluxes from SPEX. The last column shows the means and standard deviations of all 5 imaging methods.

\begin{tabular}{lrrrrrr}
\hline $\begin{array}{l}\text { Energy } \\
\text { range }\end{array}$ & $\begin{array}{r}\text { Forward } \\
\text { flux ratio }\end{array}$ & $\begin{array}{r}\text { Pixon } \\
\text { flux ratio }\end{array}$ & $\begin{array}{r}\text { Clean } \\
\text { flux ratio }\end{array}$ & $\begin{array}{r}\text { MEM } \\
\text { flux ratio }\end{array}$ & $\begin{array}{r}\text { MEM-Vis } \\
\text { flux ratio }\end{array}$ & $\begin{array}{r}\text { Average } \\
\text { flux ratio }\end{array}$ \\
\hline $10-12$ & 403.41 & 378.50 & 369.52 & 358.38 & 303.28 & $362.62 \pm 37.09$ \\
$15-17$ & 56.72 & 55.76 & 55.20 & 50.79 & 50.16 & $53.72 \pm 3.02$ \\
$20-22$ & 18.99 & 18.96 & 19.13 & 17.21 & 17.70 & $18.40 \pm 0.88$ \\
$25-29$ & 15.76 & 15.38 & 16.22 & 14.16 & 14.84 & $15.27 \pm 0.80$ \\
$30-34$ & 8.56 & 8.25 & 8.54 & 7.85 & 7.29 & $8.10 \pm 0.54$ \\
$35-39$ & 5.19 & 5.33 & 5.38 & 9.60 & 4.74 & $6.05 \pm 2.00$ \\
$40-50$ & 7.01 & 6.80 & 7.31 & 6.33 & 6.59 & $6.81 \pm 0.38$ \\
$50-60$ & 3.49 & 3.25 & 3.51 & 3.14 & 2.91 & $3.26 \pm 0.25$ \\
\hline $10-12$ & 0.98 & 0.92 & 0.90 & 0.87 & 0.74 & $0.88 \pm 0.09$ \\
$15-17$ & 1.02 & 1.01 & 0.99 & 0.92 & 0.90 & $0.97 \pm 0.05$ \\
$20-22$ & 1.03 & 1.03 & 1.04 & 0.93 & 0.96 & $1.00 \pm 0.05$ \\
$25-29$ & 1.05 & 1.02 & 1.08 & 0.94 & 0.98 & $1.01 \pm 0.05$ \\
$30-34$ & 1.04 & 1.00 & 1.04 & 0.95 & 0.89 & $0.99 \pm 0.07$ \\
$35-39$ & 1.02 & 1.04 & 1.06 & 1.88 & 0.93 & $1.19 \pm 0.39$ \\
$40-50$ & 1.11 & 1.08 & 1.16 & 1.01 & 1.05 & $1.08 \pm 0.06$ \\
$50-60$ & 0.98 & 0.91 & 0.99 & 0.88 & 0.82 & $0.92 \pm 0.07$ \\
\hline Average & 1.03 & 1.00 & 1.03 & 1.05 & 0.91 & \\
& \pm 0.04 & \pm 0.06 & \pm 0.08 & \pm 0.34 & \pm 0.10 & \\
\hline
\end{tabular}

input in form of time-tagged photon event lists, but the RESSI response function is applied by independent modules to obtain photon counts in a given energy range. A first basic photometry test that verifies the consistency of the software is therefore to compare the photon counts of the SPEX spectra in different energy ranges with those obtained from images in the same energy ranges. The time profile of the flare, the photon spectrum, the photon count rates and pre-flare background in 8 energy ranges are all shown in $\S \mathrm{A} 1$ on the CD-ROM. The pre-flare background amounts to a few percent of the peak flux (at energies $\lesssim 40$ $\mathrm{keV})$.

\subsection{Image Photometry (§A2 on CD-ROM)}

We conduct a photometric test by comparing the photon rates (in units of photons $\mathrm{s}^{-1} \mathrm{~cm}^{-2}$ ) integrated over each image with those obtained from the total flux spectra calculated with SPEX in identical energy bins. We find that these total fluxes are well conserved in most of the imaging reconstruction algorithms (see ratios in Table 1), they agree 


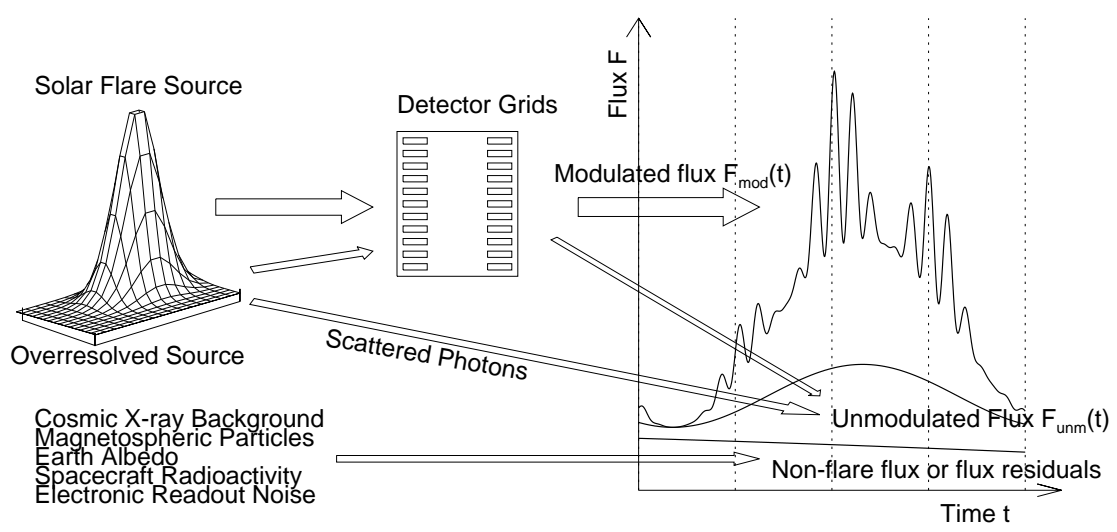

Figure 1. Schematic cartoon of solar and non-solar sources that produce modulated flux $F_{\text {mod }}(t)$, unmodulated flux $F_{u n m}(t)$, and non-flare sources $F_{b a c k}(t)$ in the time domain of the modulation time profiles.

with $S P E X$ within a few percents for all 5 imaging algorithms (Forwardfit, Pixon, Clean, MEM, MEM-Vis). The photometric accuracy seems to be uniform in different energy ranges (last column in Table 1). This test essentially verifies that the flux normalization is correctly and consistently implemented in the different software modules of each imaging algorithm.

\subsection{Background Components ( $§$ A3 on CD-ROM)}

The imaged flux consists of at least 3 different components that can be distinguished in the image reconstruction process: (1) modulated flux $F_{\text {mod }}(t)$ that is modulated by the rotating grids and originates from resolved flare sources, (2) unmodulated flux $F_{u n m}(t)$, which shows no grid modulation but varies proportionally to the smoothed flare flux, probably produced by scattered flare photons or by overresolved sources that are larger than the pitch of the coarsest grid, and (3) non-flare background flux $F_{b a c k}(t)$ that may originate from cosmic Xray background, magnetospheric particles, Earth albedo, spacecraft radioactivity, or electronic readout noise (Fig. 1). Background modeling can be performed with the Pixon and Forward-Fit algorithms. We find an (unexplained) unmodulated flux in the order of $\approx 15 \%$, in excess of the preflare background rate $(\approx 1 \%)$. Although the thin attenuator was inserted during this flare, it has probably no effect on the unmodulated flux because it is located after the grids. 


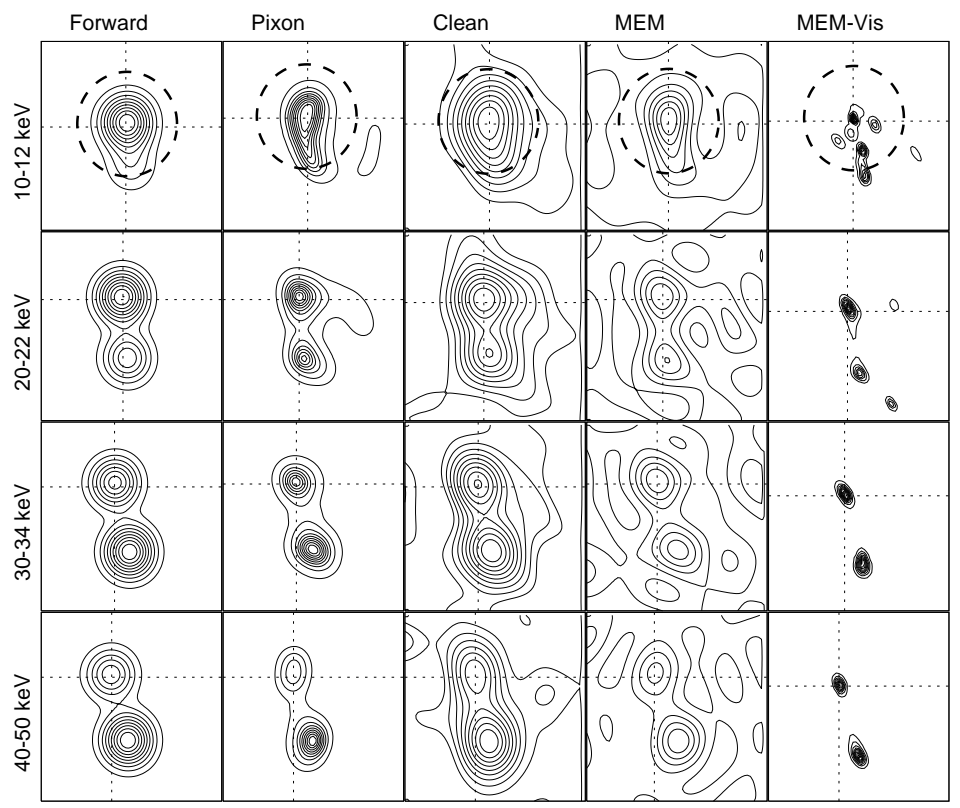

Figure 2. Image comparisons of Forward-fit, Pixon, Clean, MEM, and MEM-Vis images reconstructed in 4 energy bins. The contour levels are at 10\%, 20\%, .., $90 \%$ of the maximum flux. The location of the northern flux maximum is indicated with a dotted cross-hair. The dotted circle has a radius of 18", which is used for halo/core flux ratio measurements.

\subsection{Positional Comparisons (§A4 on CD-ROM)}

Images reconstructed in 4 energy bands with all 5 algorithms are shown in Fig. 2. The source morphology is a simple double footpoint geometry, which is consistently reproduced by all 5 algorithms. MEM-Vis in its current implementation does not take corrections for time variability into account, and thus seems to have a compromised image quality for this case (integrated over a time interval of $40 \mathrm{~s}$ ). We measure the centroid location $\left(x_{N}, y_{N}\right)$ of the northern flare source with sub-pixel accuracy by parabolic interpolation at the source peak. Averaging these source positions among the different image algorithms we find a standard deviation in the order of 0.5 pixels (with pixel size of 1 "), which is close to the accuracy expected from simulations for the corresponding count rates. Only MEM-Vis shows occasionally larger deviations, very likely to be a side-effect of the compromised image quality. 


\subsection{Amplitude and Width Comparisons (§A5 on CD-ROM)}

The reconstructed source widths $w$ vary to a larger degree between individual imaging algorithms. Compared with the spatial resolution of the finest used grid $\left(w_{\text {res }} \approx 11.8\right.$ " for grid $\left.\# 4\right)$, we find that the width ratio of the reconstructed nonthermal sources at $25 \mathrm{keV}$ is $w / w_{\text {res }}=1.2$ for Forward-Fit, $w / w_{\text {res }}=0.7$ for Pixon, $w / w_{\text {res }}=1.7$ for Clean, $w / w_{\text {res }}=3.3$ for MEM, and $w / w_{\text {res }}=0.2$ for MEM-Vis. The Pixon and Forward-Fit algorithms yield compatible widths, while those from $M E M$ are significantly larger, and those from MEM-Vis are systematically narrower (probably a side-effect of compromised image quality for time-variable sources). The larger widths obtained from Clean maps result from the convolution with the Clean beam. Apparently, MEM does not converge to the best solution when there are too many degrees of freedom.

The peak flux amplitude $a$ of an image map is roughly reciprocal to the square of the source widths, i.e.m $a \propto 1 / w^{2}$, because the flux per feature $\left(F \approx a w^{2}\right)$ is well-conserved. Because of this strong sensitivity to the reconstructed source width, it is not unusual that the peak flux differs up to an order of magnitude between different image algorithms, depending on the convergence behavior of the reconstruction algorithm. For imaging spectroscopy, therefore, it is important to sum over an entire feature (such as a flare footpoint) rather than to examine the flux of a source on a pixel by pixel basis.

\subsection{Halo/Core Flux Ratios ( $\S$ A6 on CD-ROM)}

We measure the flux ratio between a core $(C)$ region (inside a radius of 1.5 the resolution of the finest grid) and the outer halo $(H)$ region. This $\mathrm{H} / \mathrm{C}$ flux ratio reflects how many photons are spread in the image outside the real sources. It is found to be low for Forward-fit, Pixon, and Mem-Vis, in the order of a few percent, while Clean shows a somewhat larger ratio of $H / C \lesssim 30 \%$ due to the convolution with the clean beam and the addition of clean residuals. MEM exhibits a large noise ratio of $H / C \lesssim 160 \%$, a clear indication that the image algorithm does not converge properly in some cases, in particular for small pixel sizes (1") and large field-of-views, which corresponds to a larger degree of freedom in the image reconstruction.

\subsection{Spatially Resolved Spectra (§A7 on CD-ROM)}

We fit a two-component (thermal plus non-thermal powerlaw) spectrum to the image fluxes obtained in 8 different energy channels. Comparisons of the best-fit parameters with those obtained with the spec- 
tral fitting software SPEX show agreement between the non-thermal fluxes and power-law slopes within a few percents for all imaging algorithms. However, we emphasize that spatially-resolved spectra should only be extracted from images by integrating over an entire source structure (or image feature), never from single pixels (because of the amplitude-width uncertainty, see $\S 2.5)$.

\subsection{Grid Selection ( $§ \mathrm{~A} 8$ on CD-ROM)}

The grid selection determines the width of the reconstructed sources in the case of unresolved sources. We found that the reconstructed source width indeed scales (within a factor of 2) with the spatial resolution of the finest used grid for most of the image algorithms (except for MEM-Vis, which over-resolves, and for MEM, which under-resolves the sources).

\subsection{Pixel-size Comparisons ( $§$ A9 on CD-ROM)}

The image reconstruction should provide a solution that is independent of the map parameterization, such as the field-of-view or pixel size, provided that the sources are resolved. Only in the case of unresolved sources, the reproduced source width represents an upper limit specified by the pixel size. We reconstruct the images with pixel sizes of 1", 2", and 4" and find indeed an invariant solution for 4 algorithms, with a width that is related to the resolution of the finest used grid. Only MEM-VIS shows a different behavior, yielding a source size that scales with the pixel size rather than with the resolution of the finest used grid.

\subsection{Field-OF-View Comparisons (§A10 on CD-ROM)}

The selected field-of-view is found not to affect the quality of the image reconstruction for Forward-fit, Pixon, and Clean. For MEM and $M E M-V i s$, the quality of the image reconstruction seems to improve for minimal field-of-views that closely encompass the sources, apparently because this minimizes the number of degrees of freedom in the image reconstruction.

\subsection{Time Interval Comparisons (§A11 on CD-ROM)}

Since the time interval is proportional to the number of photons used in the image reconstruction, the quality and signal-to-noise ratio is expected to improve for longer time intervals. We find that the signalto-noise ratio indeed improves with longer time intervals for Pixon, 
Clean, and MEM-Vis. Forward-fit is a noise-free model by definition, so that a noise-to-signal ratio is not defined. For MEM we find no noise-to-signal improvement for longer time intervals, when the number of degrees of freedom are too large (for small pixel sizes and large field-of-views).

\section{CONCLUSIONS}

This study demonstrates that most available RHESSI image algorithms have a reasonable photometric accuracy for the total flux contained in the images, but differ substantially in the geometry of the reconstructed sources. The results can be summarized as follows: Three algorithms (Forward-Fit, Pixon, Clean) converge to the best solution within the constraints of the spatial resolution of the finest used grids, within the expected uncertainty of the signal-to-noise dictated by photon statistics. The current implementation of the MEM algorithm does not converge well for cases with a large number of degrees of freedom (for small pixel sizes and large field-of-views). The current version of MEM-Vis does not correct for time variability and thus yields a compromised image quality for long-duration time intervals. Nevertheless, the user can produce consistent and high-quality images with the $M E M$ and MEM-Vis algorithms by exercising a careful choice of image parameters.

Acknowledgements: We acknowledge constructive and critical comments from David Smith and Richard Schwartz. Support for this work was provided by the NASA SMEX grant NAS5-98033 through University of California, Berkeley (subcontract SA2241-26308PG).

\section{References}

Alexander,D. and Metcalf,T.R. 1997, Astrophys.J. 489, 442.

Aschwanden,M.J., Schmahl,E., and RHESSI Team, 2002a, Solar Physics 210, 193. Aschwanden,M.J., Brown,J.C., and Kontar,E.P., 2002b, Solar Physics 210, 383.

Brown,J.C., Aschwanden,M.J. and Kontar,E.P., 2002, Solar Physics 210, 373.

Hurford,G.J., Schmahl,E.J., Schwartz,R.A., Conway,A.J., Aschwanden,M.J., Csillaghy,A., Dennis,B.R., Johns-Krull,C., Krucker,S., Lin,R.P., McTiernan,J., Metcalf,T.R., Sato,J., and Smith,D.M. 2002, Solar Physics 210, 61.

Kontar,E.P., Brown,J.C., and McArthur,G.K. 2002, Solar Physics, 210, 419.

Krucker,S. and Lin,R.P. 2002, Solar Physics, 210, 229.

Lin,R.P. and 65 co-authors 2002, Solar Physics 210, 3.

Metcalf,T.R., Hudson,H.S., Kosugi,T., Puetter,R.C., Pina,R.K. 1996, ApJ 466, 585.

Smith,D.M. et al. 2002, Solar Physics 210, 33. 
Schwartz,R.A., Csillaghy,A., Tolbert,A.K., Hurford,G.J.,McTiernan,J., and Zarro,D. 2002, Solar Physics 210, 165.

Sui,L., Holman,G.D., Dennis,B.R., Krucker,S., Schwartz,R.A., and Tolbert,K. 2002, Solar Physics 210, 245.

Vilmer,N., Krucker,S., Lin,R.P., and RHESSI Team 2002, Solar Physics 210, 261. 
photo.tex; 30/10/2018; $19: 25 ;$ p.10 